\title{
Comparative analysis of Mycobacterium abscessus clinical isolate virulence using an invertebrate infection model
}

Yasuhiko Matsumoto, ${ }^{1, *}$, Hanako Fukano², Naoki Hasegawa³, Yoshihiko Hoshino ${ }^{2, *}$, Takashi Sugita $^{1}$

${ }^{1}$ Department of Microbiology, Meiji Pharmaceutical University, Tokyo, 204-8588, Japan

${ }^{2}$ Department of Mycobacteriology, Leprosy Research Center, National Institute of Infectious

Diseases, Tokyo, 189-0002, Japan

${ }^{3}$ Department of Infectious Diseases, Keio University School of Medicine, Tokyo, 160-8582, Japan

Abbreviated running headline: Mycobacterium abscessus virulence

${ }^{*}$ Corresponding authors: Dr. Yasuhiko Matsumoto, Department of Microbiology, Meiji

Pharmaceutical University, 2-522-1, Noshio, Kiyose, Tokyo 204-8588, Japan, Tel: +81-42-495-

8745, e-mail: ymatsumoto@my-pharm.ac.jp; Dr. Yoshihiko Hoshino, Department of Mycobacteriology, Leprosy Research Center, National Institute of Infectious Diseases, 4-2-1 Aoba, Higashi-Murayama, Tokyo 189-0002, Japan, e-mail: yhoshino@niid.go.jp. 


\begin{abstract}
Aims: Mycobacterium abscessus causes chronic skin infections, lung diseases, and systemic or disseminated infections. We investigated the quantitative evaluation of the virulence of $M$. abscessus clinical isolates by calculating the $\mathrm{LD}_{50}$ using a silkworm infection model.

Methods and Results: M. abscessus subsp. abscessus cells were injected into the hemolymph of silkworms. Silkworms died within two days post-infection with M. abscessus subsp. abscessus when reared at $37^{\circ} \mathrm{C}$. Viable cell numbers of M. abscessus subsp. abscessus increased in the hemolymph of the silkworms injected with M. abscessus subsp. abscessus. Silkworms were not killed following injections with heat-killed M. abscessus subsp.abscessus cells. The administration of clarithromycin, an antibacterial drug used for the treatment of the infection, prolonged the survival time of silkworms injected with $M$. abscessus subsp. abscessus. The LD $_{50}$ values of seven clinical isolates were determined using the silkworm infection model and differed by up to nine-fold.
\end{abstract}

Conclusions: $M$. abscessus proliferation is required to kill the silkworms and that the virulence of M. abscessus clinical isolates can be evaluated by the silkworm infection model.

Significance and Impact of the Study: The silkworm infection model with M. abscessus is useful for estimating the virulence of the clinical isolates in a short period.

\title{
Keywords
}

Virulence, quantitative evaluation, Mycobacterium abscessus subsp.abscessus, silkworm, infection 


\section{Introduction}

The Mycobacterium abscessus complex (MABC) is a group of rapid-growing, non-tuberculous mycobacteria (NTM) that includes three subspecies: M. abscessus subsp. abscessus, $M$. abscessus subsp. massiliense, and M. abscessus subsp. bolletii (Hoshino and Suzuki, 2015; Oren and Garrity, 2016; Gupta et al., 2018; Tortoli et al., 2018). Because the MABC causes chronic skin infections and lung diseases in immunocompetent patients, and also systemic and/or disseminated infections in immunocompromised patients (Catherinot et al., 2007; Piersimoni and Scarparo, 2008; Esther et al., 2010; Johansen et al., 2020), the patients infected with these subspecies will show heterogenous clinical outcomes, suggesting the different subspecies present various degrees of virulence (Koh et al., 2011; Morimoto et al., 2018). Moreover, the virulence of M. abscessus has evolved through stepwise adaptation of host and soil environments (Catherinot et al., 2007; Bryant et al., 2021), which means that it likely varies between clinical isolates. Several mouse infection models with M. abscessus were established for evaluating the effects of antibacterial drugs (Obregón-Henao et al., 2015; Maggioncalda et al., 2020). In mouse infection models, since a long time is required to mouse death by the infection of M. abscessus (Catherinot et al., 2007), the development of a rapid evaluation system is desired.

Silkworm, an invertebrate, is a beneficial experimental animal for revealing the hostpathogen interactions (Matsumoto, 2020; Kaito et al., 2020; Montali et al., 2020). A large number of silkworms can be reared in a small space compared with mammalian animals (Matsumoto and Sekimizu, 2019). The 3Rs, replacement, refinement, and reduction, are important principles for experiments using mammals in the view of animal welfare (Herrmann et al., 2019). Experiments with invertebrates fit the concept of replacement. Since silkworm is invertebrate, few ethical issues cause by sacrificing a large number of silkworms compared with mammals. Exploiting the benefits 
of silkworms to infectious disease research, the $\mathrm{LD}_{50}$, which is the dose of a pathogen required to kill half of the silkworms in a group, can be determined for quantitatively comparing the virulence of strains (Matsumoto et al., 2011; M. Ishii et al., 2017). Silkworm infection models were used as the evaluation systems for first screening to identify virulence-related genes in pathogenic microorganisms (Kaito et al., 2005; Hanaoka et al., 2008; Ueno et al., 2011; Paudel et al., 2020). Therefore, silkworm infection models are useful for the comparing virulence of microorganisms. A silkworm infection model was established for the evaluation of anti-mycobacterial compounds using a type strain (Hosoda et al., 2020). However, comparative analysis of virulence of clinical isolates in M. abscessus based on the determination of the $\mathrm{LD}_{50}$ values was not demonstrated.

In the present study, we focused on evaluating the virulence of $M$. abscessus subsp. abscessus. We attempted to compare the virulence of $M$. abscessus subsp. abscessus clinical isolates using a systemic infection model using silkworms. We established a silkworm infection model to determine the $\mathrm{LD}_{50}$ values of $M$. abscessus subsp. abscessus isolates to quantitatively evaluate their virulence. Among the seven clinical isolates, the difference in virulence varied up to nine-fold. The results suggest that the silkworm infection model is a rapid evaluation system for quantitatively estimating the virulence of $M$. abscessus subsp. abscessus clinical isolates.

\section{Materials and Methods}

\section{Reagents}

Clarithromycin (Tokyo Chemical Industry Co., Ltd., Tokyo, Japan) was suspended in $0.9 \% \mathrm{NaCl}$ solution (saline). Middlebrook 7H9 broth, Middlebrook 7H10 agar, and Middlebrook OADC enrichment were purchased from Becton, Dickinson, and Company (MD, USA). Middlebrook 7H9 
broth and Middlebrook 7H10 agar were supplemented with 10\% Middlebrook OADC enrichment.

\section{Bacterial strains and growth}

The M. abscessus subsp. abscessus ATCC19977 strain and seven clinical isolates (Mb-7, Mb-10, $\mathrm{Mb}-14, \mathrm{Mb}-16, \mathrm{Mb}-17, \mathrm{Mb}-18$, and $\mathrm{Mb}-22)$ were used in this study. The clinical isolates were obtained from the Keio University School of Medicine. This study was approved by the medical research ethics committee of the National Institute of Infectious Diseases (\#1046) and by Keio University School of Medicine Ethics Committee (\#2008-0131-9 sai). Species were identified with a DDH Mycobacteria Kit (Kyokuto Pharmaceutical Industrial Co., Tokyo, Japan) (Kusunoki et al., 1991) and multiplex PCR (Yoshida et al., 2021). The M. abscessus subsp. abscessus strains were grown on Middlebrook $7 \mathrm{H} 10$ agar plate at $37^{\circ} \mathrm{C}$. A single colony was then inoculated into $5 \mathrm{ml}$ of Middlebrook $7 \mathrm{H} 9$ broth and incubated at $37^{\circ} \mathrm{C}$ for three days.

\section{Infection experiments using silkworms}

The silkworm infection experiments were performed as previously described (Kaito et al., 2002). Fifth instar larvae were reared with an artificial diet Silkmate 2S (Ehime-Sanshu Co., Ltd., Ehime, Japan) for 24 h. M. abscessus subsp. abscessus cells grown in Middlebrook 7H9 broth were collected by centrifugation and suspended in sterile saline. Silkworms injected with the $M$. abscessus subsp. abscessus cells were incubated at $27^{\circ} \mathrm{C}$ or $37^{\circ} \mathrm{C}$ and their survival monitored.

Therapeutic activity tests using the silkworms were performed according to a previous study with slight modifications (Kaito et al., 2002). Either $50 \mu \mathrm{l}$ of saline or $50 \mu \mathrm{l}$ of an M. abscessus subsp. abscessus suspension $\left(6.3 \times 10^{7}\right.$ cells $)$ was injected into the silkworm hemolymph. Immediately following inoculation of the silkworm with the M. abscessus subsp. abscessus 
suspension, clarithromycin was injected the silkworms at a concentration of $25 \mu \mathrm{g} \mathrm{g}^{-1}$ larva.

\section{Viable cell counts}

Silkworms were injected with an M. abscessus subsp. abscessus cell suspension $\left(7 \times 10^{6}\right.$ cells in $50 \mu \mathrm{l})$ and incubated at $37^{\circ} \mathrm{C}$. Hemolymph was harvested from the silkworm larva at either 3 or 30 $\mathrm{h}$ post-infection through a cut on the first proleg (Matsumoto et al., 2011). The hemolymph was added to saline and the solution was spread on a Middlebrook 7H10 agar plate. The agar plate was incubation at $37^{\circ} \mathrm{C}$ for 3 days, the colonies on the agar plate were counted. The total number of viable cells in the sample was calculated.

\section{LD $_{50}$ measurement}

$\mathrm{LD}_{50}$ values were determined according to a previous study, with slight modifications (Matsumoto et al., 2012; Miyazaki et al., 2012). M. abscessus subsp. abscessus cells grown in Middlebrook 7H9 broth were suspended in saline. Either a two- or four-fold dilution series of the bacterial suspension was prepared, followed by $50 \mu 1$ injections into silkworm hemolymph $\left(4 \mathrm{x} 10^{5}-1 \mathrm{x}\right.$ $10^{8}$ cells), and incubation at $37^{\circ} \mathrm{C}$. The survival number of silkworms was observed at $48 \mathrm{~h}$. The $\mathrm{LD}_{50}$ values were determined from the data of three experiments using a simple logistic regression model in Prism 9 (GraphPad Software, LLC, San Diego, CA, USA, https://www.graph pad.com/scientific-software/prism/).

\section{Statistical test}

The statistical significance of differences between the viable cell counts of $M$. abscessus subsp. abscessus in silkworm groups were determined by the Student $t$-test. 


\section{Results}

Experimental conditions for the evaluation of $M$. abscessus subsp. abscessus virulence in silkworms

We first determined the experimental conditions for evaluating the virulence of $M$. abscessus subsp. abscessus in silkworms. The rearing temperature in a silkworm infection experiment is critical because it regulates the body temperature that affects bacterial virulence (Matsumoto and Sekimizu, 2019). Silkworms were died within 48 h by injection of M. abscessus subsp. abscessus ATCC19977 strain $\left(1.6 \times 10^{9}\right.$ cells) at $37^{\circ} \mathrm{C}$, but not died at $27^{\circ} \mathrm{C}$ (Fig. 1). The $\mathrm{LD}_{50}$ for $M$. abscessus subsp. abscessus ATCC19977 was $1.1 \times 10^{7}$ cells under a $37^{\circ} \mathrm{C}$ incubating condition (Fig. 2). These results suggest that $37^{\circ} \mathrm{C}$ condition is necessary for M. abscessus subsp. abscessusinduced silkworm death that can be evaluated within two days.

\section{Effect of M. abscessus subsp. abscessus proliferation in silkworms on virulence}

Since the silkworm death caused by Porphyromonas gingivalis in a previous report did not require proliferation, we hypothesized that to be a result of a shock, and not a bacterial infection (K. Ishii et al., 2010). To evaluate that possibility for M. abscessus subsp. abscessus in our model, we evaluated if proliferation was necessary for virulence (see the experimental schematic in Fig. 3A). The M. abscessus subsp. abscessus viable cell count increased in the silkworm hemolymph at 30 h post-infection (Fig. 3B). The injection of autoclaved M. abscessus subsp. abscessus cells, however, did not kill silkworms (Fig. 4A). The administration of clarithromycin, an antibiotic clinically used for M. abscessus subsp. abscessus infections, to silkworms post-infection with $M$. abscessus subsp. abscessus prolonged their survival time (Fig. 4B). These results suggest that $M$. 
abscessus subsp. abscessus virulence in silkworms requires M. abscessus subsp. abscessus growth.

\section{Comparative pathogenic analysis of M. abscessus subsp. abscessus clinical isolates}

We next determined the $\mathrm{LD}_{50}$ values of $M$. abscessus subsp. abscessus clinical isolates using the silkworm infection model to compare their virulence. Seven clinical isolates were obtained from sputum samples of patients infected with M. abscessus subsp. abscessus. Their $\mathrm{LD}_{50}$ values ranged from $3.1 \times 10^{6}$ to $2.9 \times 10^{7}$ cells per larva; the $\mathrm{LD}_{50}$ value of the $\mathrm{Mb}-17$ isolate was the lowest (Fig. 5). The $\mathrm{LD}_{50}$ value of the Mb-17 isolate was nine-fold lower than that of the Mb-10 isolate (Fig. 5). These results suggest that the Mb-17 isolate is highly pathogenic to silkworms compared to the other isolates.

\section{Discussion}

In the present study, the virulence of $M$. abscessus subsp. abscessus clinical isolates was compared using a silkworm infection model. Among the seven clinical isolates, the virulence, as determined by the $\mathrm{LD}_{50}$, varied by up to nine-fold. The results indicate that the in vivo evaluation system is useful for revealing the virulence of M. abscessus subsp. abscessus clinical isolates in a shorter period of time (within two days).

M. abscessus subsp. abscessus-infected silkworms incubated at $37^{\circ} \mathrm{C}$, human body temperature, were more sensitive to infection than those reared at $27^{\circ} \mathrm{C}$, the normal rearing temperature of silkworms. We assumed that the difference was caused by both high-temperature stress in silkworms and the optimal growth temperature for M. abscessus subsp. abscessus. Hosoda et. al. reported to establish a silkworm infection model for evaluating the anti-mycobacterial compounds (Hosoda et al., 2020). Compared to that report, we demonstrated that M. abscessus 
subsp. abscessus grows in silkworm hemolymph and evaluated the virulence of several clinical isolates. Our findings are important for validating the usefulness of silkworm to estimate the $M$. abscessus subsp. abscessus clinical isolate virulence.

M. abscessus subsp. abscessus virulence may correlate with severe infections (Catherinot et al., 2007; Bryant et al., 2021). Therefore, understanding the M. abscessus subsp. abscessus clinical isolate virulence is information for infection control. M. abscessus subsp. abscessus showed different virulence among clinical isolates in the silkworm model. We demonstrated that the silkworm infection model with M. abscessus subsp. abscessus is a beneficial evaluation system that enables a quantitative determination of clinical isolate virulence by calculating the $\mathrm{LD}_{50}$ values within just two days. Moreover, the $\mathrm{LD}_{50}$ values among the clinical isolates were differed by up to nine-fold. Mb-17 was the most pathogenic isolate in silkworms among the M. abscessus subsp. abscessus clinical isolates used in this study; it may harbor virulence-related genes that enhance the infection process. The virulence genes of several pathogens were identified by avirulent mutant screening using silkworm infection models from a mutant library (Kaito et al., 2005; Hanaoka et al., 2008; Ueno et al., 2011; Paudel et al., 2020). The method for constructing M. abscessus subsp. abscessus gene-deletion mutants is well established (Küssau et al., 2020; Foreman et al., 2020). Further studies are needed to determine the virulence factors that the $M$. abscessus subsp. abscessus $\mathrm{Mb}-17$ isolate harbours that are responsible for its virulence in silkworms.

M. abscessus vertebrate infection models using a zebrafish, Danio rerio, and a tadpole, Xenopus laevis, were reported (Küssau et al., 2020; Kim et al., 2021; Lopez et al., 2021). These infection models are needed for the evaluation of anti-mycobacterial drugs and the virulence within 15 days. Since these model animals are vertebrates, ethical problems and protocols must be 
managed to ensure animal welfare. The 3Rs, replacement, refinement, and reduction, are important principles for experiments using mammals in the view of animal welfare (Herrmann et al., 2019). Silkworms are invertebrates, which have merits as an alternative animal for infection experiments requiring large groups. Moreover, M. abscessus subsp. abscessus virulence factors, which are expressed in humans, might be identified by the silkworm infection model with $37^{\circ} \mathrm{C}$ conditions.

We propose that the silkworm infection model with M. abscessus subsp. abscessus is a beneficial assay system for determining the virulence of $M$. abscessus subsp. abscessus clinical strains. The silkworm infection model may contribute to revealing the molecular mechanisms $M$. abscessus subsp. abscessus infections.

\section{Author's contribution}

YM designed the study, performed experiments, collected data, and wrote the initial draft of the manuscript. FH, NH, and YH prepared resources such as the M. abscessus subsp. abscessus clinical isolates. $\mathrm{YH}, \mathrm{FH}$, and TS contributed to data interpretation and critically reviewed the manuscript. All authors read and approved the final manuscript.

\section{Acknowledgements}

We thank Tae Nagamachi, Asami Yoshikawa, Yu Sugiyama, Eri Sato, and Asuka Toshima (Meiji Pharmaceutical University) for their technical assistance rearing the silkworms. We also thank Maki Okuda, Sayaka Kashiwagi, and Ginko Kaneda for their assistance. This study was supported in part by grants from the Japan Agency for Medical Research and Development/Japan International Cooperation Agency (AMED) to Y.H. (jp20fk0108064, jp20fk0108075, jp21fk0108093， jp21fk0108129， jp21fk0108608， jp21jm0510004， jp21wm0125007, 
jp21wm0225004, and jp21wm0325003); by grants-in-aid for Fostering Joint International Research (B) to Y.H. (jp19KK0217) and for Early-Career Scientists to H.F. (jp18K15966); by Grants-in-Aid for Scientific Research (B) to Y.H. (jp20H02282); and for Scientific Research (C) to Y.M. (JP20K07022) from the Japan Society for the Promotion of Science (JSPS). The funders had no role in the study design, data collection, data analysis, the decision to publish, or preparation of the manuscript.

\section{Conflict of interest}

The authors declare no conflict of interest.

\section{Data availability statement}

All data identified in the present study are provided in the paper and its supplementary information (S1 Dataset). 


\section{References}

Bryant, J.M., Brown, K.P., Burbaud, S., Everall, I., Belardinelli, J.M., Rodriguez-Rincon, D., et al. (2021) Stepwise pathogenic evolution of Mycobacterium abscessus. Science 372: 6541.

Catherinot, E., Clarissou, J., Etienne, G., Ripoll, F., Emile, J.-F., Daffé, M., et al. (2007) Hypervirulence of a rough variant of the Mycobacterium abscessus type strain. Infect. Immun. 75: 1055-1058.

Esther, C.R., Esserman, D.A., Gilligan, P., Kerr, A., and Noone, P.G. (2010) Chronic Mycobacterium abscessus infection and lung function decline in cystic fibrosis. J Cyst Fibros 9: $117-123$.

Foreman, M., Gershoni, M., and Barkan, D. (2020) A Simplified and Efficient Method for Himar1 Transposon Sequencing in Bacteria, Demonstrated by Creation and Analysis of a Saturated Transposon-Mutant Library in Mycobacterium abscessus. mSystems 5: 5.

Gupta, R.S., Lo, B., and Son, J. (2018) Phylogenomics and Comparative Genomic Studies Robustly Support Division of the Genus Mycobacterium into an Emended Genus Mycobacterium and Four Novel Genera. Front Microbiol 9: 67.

Hanaoka, N., Takano, Y., Shibuya, K., Fugo, H., Uehara, Y., and Niimi, M. (2008) Identification of the putative protein phosphatase gene PTCl as a virulence-related gene using a silkworm model of Candida albicans infection. Eukaryotic Cell 7: 1640-1648.

Herrmann, K., Pistollato, F., and Stephens, M.L. (2019) Beyond the 3Rs: Expanding the use of human-relevant replacement methods in biomedical research. ALTEX 36: 343-352.

Hoshino, Y. and Suzuki, K. (2015) Differential diagnostic assays for discriminating mycobacteria, especially for nontuberculous mycobacteria: what does the future hold? Future Microbiol 10: 205-216. 
Hosoda, K., Koyama, N., Hamamoto, H., Yagi, A., Uchida, R., Kanamoto, A., and Tomoda, H. (2020) Evaluation of Anti-Mycobacterial Compounds in a Silkworm Infection Model with Mycobacteroides abscessus. Molecules 25: 4971.

Ishii, K., Hamamoto, H., Imamura, K., Adachi, T., Shoji, M., Nakayama, K., and Sekimizu, K. (2010) Porphyromonas gingivalis peptidoglycans induce excessive activation of the innate immune system in silkworm larvae. J. Biol. Chem. 285: 33338-33347.

Ishii, M., Matsumoto, Y., Yamada, T., Abe, S., and Sekimizu, K. (2017) An invertebrate infection model for evaluating anti-fungal agents against dermatophytosis. Sci Rep 7: 12289.

Johansen, M.D., Herrmann, J.-L., and Kremer, L. (2020) Non-tuberculous mycobacteria and the rise of Mycobacterium abscessus. Nat. Rev. Microbiol. 18: 392-407.

Kaito, C., Akimitsu, N., Watanabe, H., and Sekimizu, K. (2002) Silkworm larvae as an animal model of bacterial infection pathogenic to humans. Microb. Pathog. 32: 183-190.

Kaito, C., Kurokawa, K., Matsumoto, Y., Terao, Y., Kawabata, S., Hamada, S., and Sekimizu, K. (2005) Silkworm pathogenic bacteria infection model for identification of novel virulence genes. Mol. Microbiol. 56: 934-944.

Kaito, C., Murakami, K., Imai, L., and Furuta, K. (2020) Animal infection models using nonmammals. Microbiol. Immunol. 64: 585-592.

Kim, T., Hanh, B.-T.-B., Heo, B., Quang, N., Park, Y., Shin, J., et al. (2021) A Screening of the MMV Pandemic Response Box Reveals Epetraborole as a New Potent Inhibitor against Mycobacterium abscessus. Int J Mol Sci 22: 5936.

Koh, W.-J., Jeon, K., Lee, N.Y., Kim, B.-J., Kook, Y.-H., Lee, S.-H., et al. (2011) Clinical significance of differentiation of Mycobacterium massiliense from Mycobacterium abscessus. Am J Respir Crit Care Med 183: 405-410. 
Kusunoki, S., Ezaki, T., Tamesada, M., Hatanaka, Y., Asano, K., Hashimoto, Y., and Yabuuchi, E.

(1991) Application of colorimetric microdilution plate hybridization for rapid genetic identification of 22 Mycobacterium species. J. Clin. Microbiol. 29: 1596-1603.

Küssau, T., Van Wyk, N., Johansen, M.D., Alsarraf, H.M.A.B., Neyret, A., Hamela, C., et al. (2020)

Functional Characterization of the N-Acetylmuramyl-1-Alanine Amidase, Ami1, from Mycobacterium abscessus. Cells 9: 2410.

Lopez, A., Shoen, C., Cynamon, M., Dimitrakopoulou, D., Paiola, M., Pavelka, M.S., and Robert, J. (2021) Developing Tadpole Xenopus laevis as a Comparative Animal Model to Study Mycobacterium abscessus Pathogenicity. Int J Mol Sci 22: 806.

Maggioncalda, E.C., Story-Roller, E., Mylius, J., Illei, P., Basaraba, R.J., and Lamichhane, G. (2020) A mouse model of pulmonary Mycobacteroides abscessus infection. Sci Rep 10: 36908.

Matsumoto, Y. (2020) Facilitating Drug Discovery in Human Disease Models Using Insects. Biol. Pharm. Bull. 43: 216-220.

Matsumoto, Y. and Sekimizu, K. (2019) Silkworm as an experimental animal to research for fungal infections. Microbiol. Immunol.

Matsumoto, Y., Miyazaki, S., Fukunaga, D.H., Shimizu, K., Kawamoto, S., and Sekimizu, K. (2012) Quantitative evaluation of cryptococcal pathogenesis and antifungal drugs using a silkworm infection model with Cryptococcus neoformans. J. Appl. Microbiol. 112: 138-146.

Matsumoto, Y., Sumiya, E., Sugita, T., and Sekimizu, K. (2011) An invertebrate hyperglycemic model for the identification of anti-diabetic drugs. PLOS ONE 6: e18292.

Miyazaki, S., Matsumoto, Y., Sekimizu, K., and Kaito, C. (2012) Evaluation of Staphylococcus aureus virulence factors using a silkworm model. FEMS Microbiol. Lett. 326: 116-124. 
Montali, A., Berini, F., Brivio, M.F., Mastore, M., Saviane, A., Cappellozza, S., et al. (2020) A Silkworm Infection Model for In Vivo Study of Glycopeptide Antibiotics. Antibiotics (Basel) 9: 300 .

Morimoto, K., Nakagawa, T., Asami, T., Morino, E., Fujiwara, H., Hase, I., et al. (2018) Clinicomicrobiological analysis of 121 patients with pulmonary Mycobacteroides abscessus complex disease in Japan - An NTM-JRC study with RIT. Respir Med 145: 14-20.

Obregón-Henao, A., Arnett, K.A., Henao-Tamayo, M., Massoudi, L., Creissen, E., Andries, K., et al. (2015) Susceptibility of Mycobacterium abscessus to antimycobacterial drugs in preclinical models. Antimicrob. Agents Chemother. 59: 6904-6912.

Oren, A. and Garrity, G.M. (2016) List of new names and new combinations previously effectively, but not validly, published. Int J Syst Evol Microbiol 66: 2463-2466.

Paudel, A., Hamamoto, H., Panthee, S., Matsumoto, Y., and Sekimizu, K. (2020) Large-Scale Screening and Identification of Novel Pathogenic Staphylococcus aureus Genes Using a Silkworm Infection Model. J. Infect. Dis. 221: 1795-1804.

Piersimoni, C. and Scarparo, C. (2008) Pulmonary infections associated with non-tuberculous mycobacteria in immunocompetent patients. Lancet Infect Dis 8: 323-334.

Tortoli, E., Kohl, T.A., Brown-Elliott, B.A., Trovato, A., Cardoso-Leão, S., Garcia, M.J., et al. (2018) Mycobacterium abscessus, a taxonomic puzzle. Int J Syst Evol Microbiol 68: 467-469.

Ueno, K., Matsumoto, Y., Uno, J., Sasamoto, K., Sekimizu, K., Kinjo, Y., and Chibana, H. (2011) Intestinal resident yeast Candida glabrata requires Cyb2p-mediated lactate assimilation to adapt in mouse intestine. PLoS ONE 6: e24759.

Yoshida, M., Sano, S., Chien, J.-Y., Fukano, H., Suzuki, M., Asakura, T., et al. (2021) A novel DNA chromatography method to discriminate Mycobacterium abscessus subspecies and 
bioRxiv preprint doi: https://doi.org/10.1101/2021.11.26.470074; this version posted November 26, 2021. The copyright holder for this preprint (which was not certified by peer review) is the author/funder, who has granted bioRxiv a license to display the preprint in perpetuity. It is made available under aCC-BY 4.0 International license.

macrolide susceptibility. EBioMedicine 64: 103187. 


\section{Figure legends}

Figure 1 Effects of temperature on the virulence of $M$. abscessus subsp. abscessus ATCC19977 in silkworms.

Silkworms were injected with saline $(50 \mu \mathrm{l})$ or M. abscessus subsp. abscessus ATCC19977 cell suspension $\left(1.4 \times 10^{7}\right.$ cells per $\left.50 \mu \mathrm{l}\right)$ and incubated at (A) $27^{\circ} \mathrm{C}$ and (B) $37^{\circ} \mathrm{C} . \mathrm{n}=7$ per group.

Figure 2 Determination of the M. abscessus subsp. abscessus ATCC19977 LD $_{50}$ in silkworms. Silkworms were injected with saline $(50 \mu \mathrm{l})$ or M. abscessus subsp. abscessus ATCC19977 cell suspension $\left(4 \times 10^{5}-1 \times 10^{8}\right.$ cells per $\left.50 \mu \mathrm{l}\right)$ and incubated at $37^{\circ} \mathrm{C}$. The number of live and dead silkworms are indicated as 1 and 0 , respectively. The curve is data from six independent experiments combined in a simple logistic regression model.

Figure 3 Increase of $M$. abscessus subsp. abscessus ATCC19977 viable cell counts in silkworms.

(A) Experiment schematic. (B) Silkworms were injected with M. abscessus subsp. abscessus ATCC19977 cell suspensions $\left(7 \times 10^{6}\right.$ cells per $\left.50 \mu \mathrm{l}\right)$ and incubated at $37^{\circ} \mathrm{C}$. Silkworm hemolymph was harvested at 3 or $30 \mathrm{~h}$ post-infection. The viable number of $M$. abscessus subsp. abscessus cells in the samples was measured by counting the colony-forming units (CFU). Statistically significant differences between groups were evaluated using Student $t$-test. $\mathrm{n}=3$ per group.

Figure 4 Effects of autoclaved cells and antibacterial treatment in silkworms infected with M. abscessus subsp. abscessus 
(A) Silkworms were injected with either saline (50 $\mu 1)$, an M. abscessus subsp. abscessus cell suspension $\left(1.1 \times 10^{7}\right.$ cells per $\left.50 \mu \mathrm{l}\right)$, or an autoclaved M. abscessus subsp. abscessus cell suspension and incubated at $37^{\circ} \mathrm{C} . \mathrm{n}=10$ per group.

(B) Silkworms were injected with either saline $(50 \mu \mathrm{l})$ or an M. abscessus subsp. abscessus cell suspension $\left(6.3 \times 10^{7}\right.$ cells per $\left.50 \mu \mathrm{l}\right)$ followed by clarithromycin $\left(25 \mu \mathrm{g} \mathrm{g}^{-1}\right.$ larva). The number of surviving silkworms following incubation at $37^{\circ} \mathrm{C}$ was measured during $66 \mathrm{~h}$. Statistically significant differences between groups were evaluated using a log-rank test. $n=10$ per group.

Figure 5 Comparison of virulence among $M$. abscessus subsp. abscessus clinical isolates in a silkworm infection model

(A-C) Silkworms were injected with either saline, M. abscessus subsp. abscessus, Mb-7, Mb-10, Mb-14, Mb-16, Mb-17, Mb-18, or Mb-22 cell suspensions $\left(2 \times 10^{5}-3.5 \times 10^{7}\right.$ cells per $\left.50 \mu 1\right)$ and incubated at $37^{\circ} \mathrm{C}$. Live and dead silkworms are indicated as 1 and 0 , respectively. The curves are data from three independent experiments combined in a simple logistic regression model.

(D) A plot of $\mathrm{LD}_{50}$ values determined from A-C. 

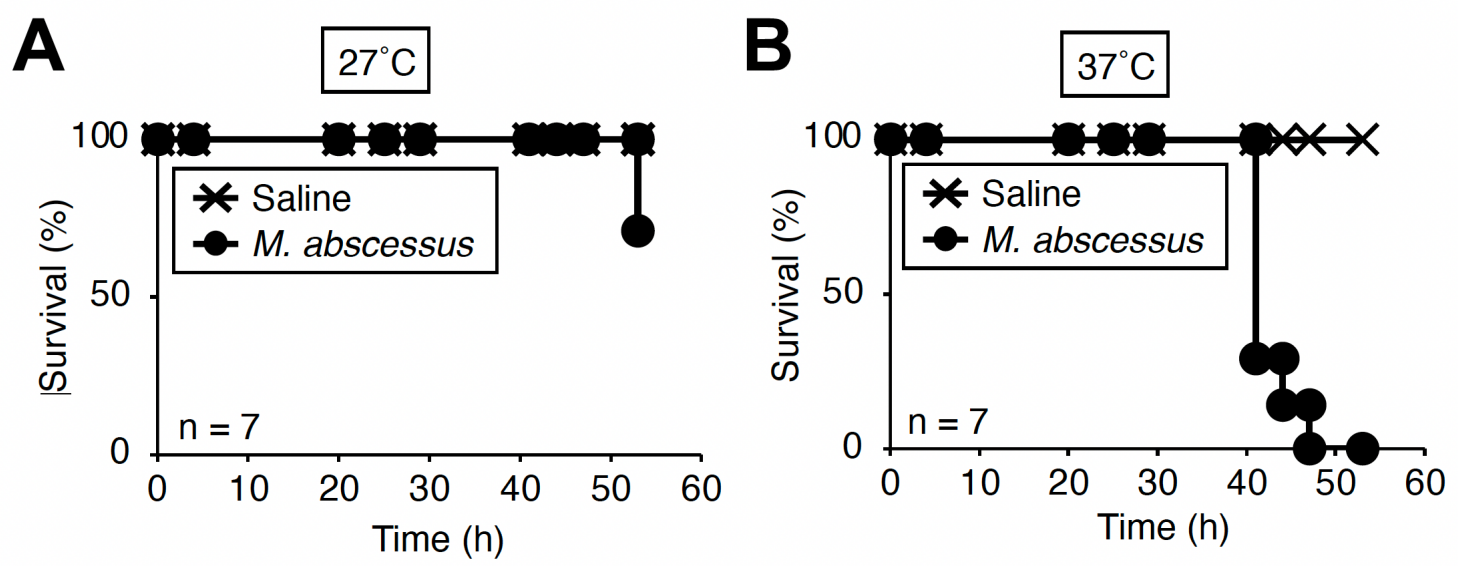

Figure 1. Effects of temperature on the pathogenicity of M. abscessus subsp. abscessus ATCC19977 in silkworms.

Silkworms were injected with saline $(50 \mu \mathrm{l})$ or $M$. abscessus subsp. abscessus ATCC19977 cell suspension $\left(1.4 \times 10^{7}\right.$ cells $\left./ 50 \mu \mathrm{l}\right)$ and incubated at $(\mathbf{A}) 27^{\circ} \mathrm{C}$ and $(\mathbf{B}) 37^{\circ} \mathrm{C} . \mathrm{n}=7 /$ group. 


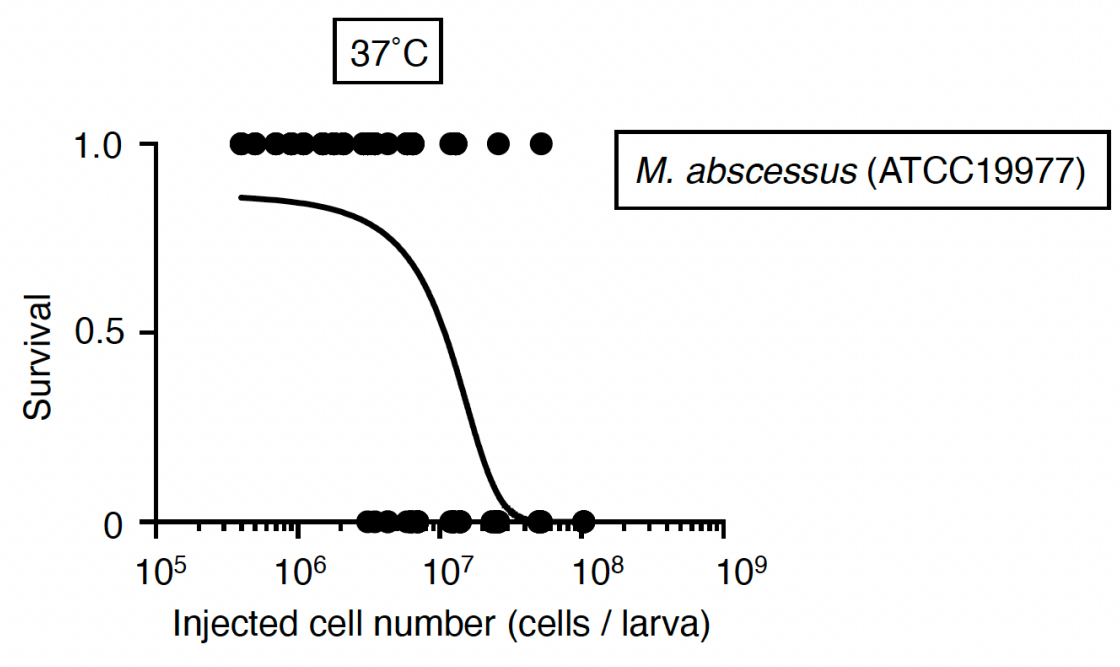

Figure 2. Determination of the $M$. abscessus subsp. abscessus ATCC19977 $\mathrm{LD}_{50}$ in silkworms.

Silkworms were injected with saline $(50 \mu \mathrm{l})$ or $M$. abscessus subsp. abscessus ATCC19977 cell suspension $\left(4 \times 10^{5}-1 \times 10^{8}\right.$ cells $\left./ 50 \mu \mathrm{l}\right)$ and incubated at $37^{\circ} \mathrm{C}$. The number of live and dead silkworms are indicated as 1 and 0 , respectively. The curve is data from six independent experiments combined in a simple logistic regression model. 


\section{A}

M. abscessus subsp. abscessus injection

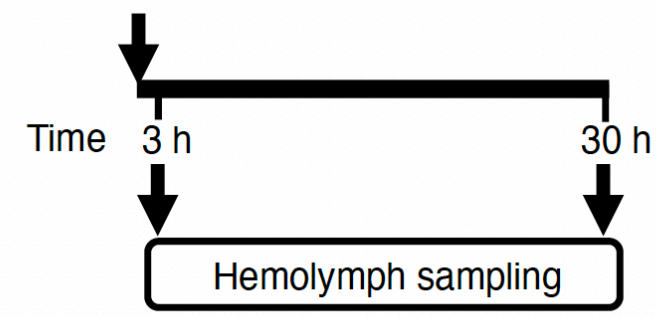

\section{B}

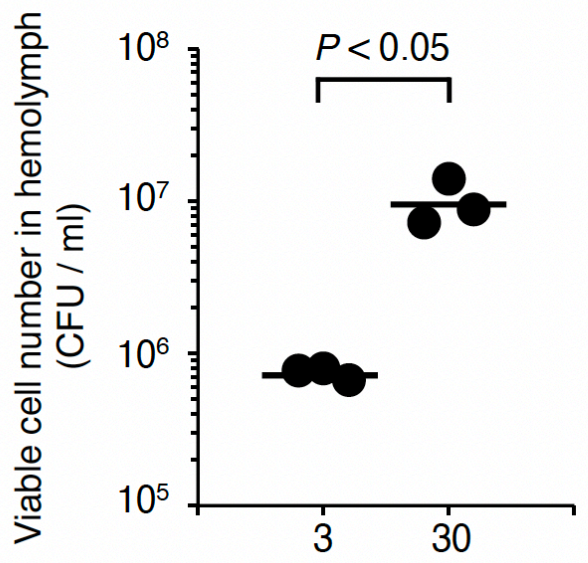

Sampling time after injection (h)

Figure 3. Increase of M. abscessus subsp. abscessus ATCC19977 viable cell counts in silkworms.

(A) Experiment schematic. (B) Silkworms were injected with $M$. abscessus subsp. abscessus ATCC19977 cell suspensions $\left(7 \times 10^{6}\right.$ cells/50 $\left.\mu \mathrm{l}\right)$ and incubated at $37^{\circ} \mathrm{C}$. Silkworm hemolymph was harvested at 3 or $30 \mathrm{~h}$ post-infection. The viable number of $M$. abscessus subsp. abscessus cells in the samples was measured by counting the colony-forming units (CFU). Statistically significant differences between groups were evaluated using Student $t$-test. $n=3 /$ group. 


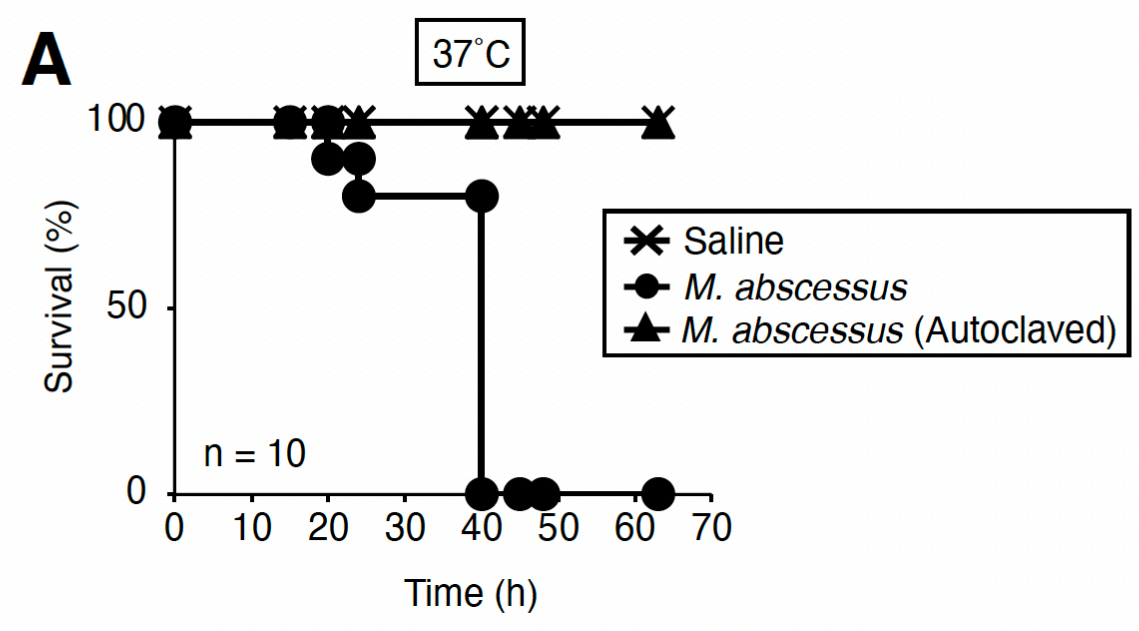

B

$37^{\circ} \mathrm{C}$

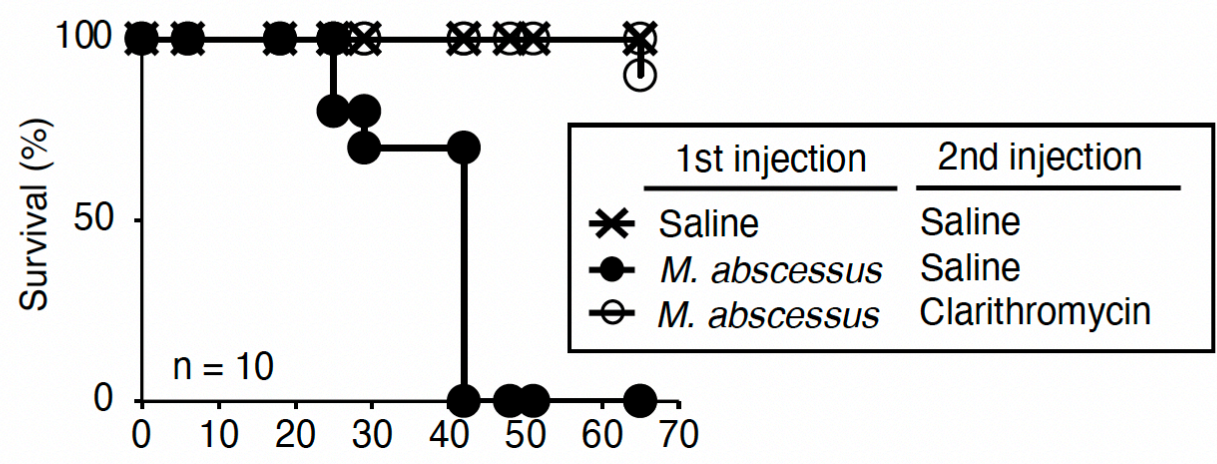

Time (h)

Figure 4. Effects of autoclaved cells and antibacterial treatment in silkworms infected with $M$. abscessus subsp. abscessus

(A) Silkworms were injected with either saline (50 $\mu \mathrm{l})$, an $M$. abscessus subsp. abscessus cell suspension $\left(1.1 \times 10^{7}\right.$ cells $\left./ 50 \mu \mathrm{l}\right)$, or an autoclaved $M$. abscessus subsp. abscessus cell suspension and incubated at $37^{\circ} \mathrm{C}$. $n=10 /$ group.

(B) Silkworms were injected with either saline $(50 \mu \mathrm{l})$ or an $M$. abscessus subsp. abscessus cell suspension $\left(6.3 \times 10^{7}\right.$ cells $\left./ 50 \mu \mathrm{l}\right)$ followed by clarithromycin (25 $\mu \mathrm{g} / \mathrm{g}$ larva). The number of surviving silkworms following incubation at $37^{\circ} \mathrm{C}$ was measured during $66 \mathrm{~h}$. Statistically significant differences between groups were evaluated using a logrank test. $n=10 /$ group. 

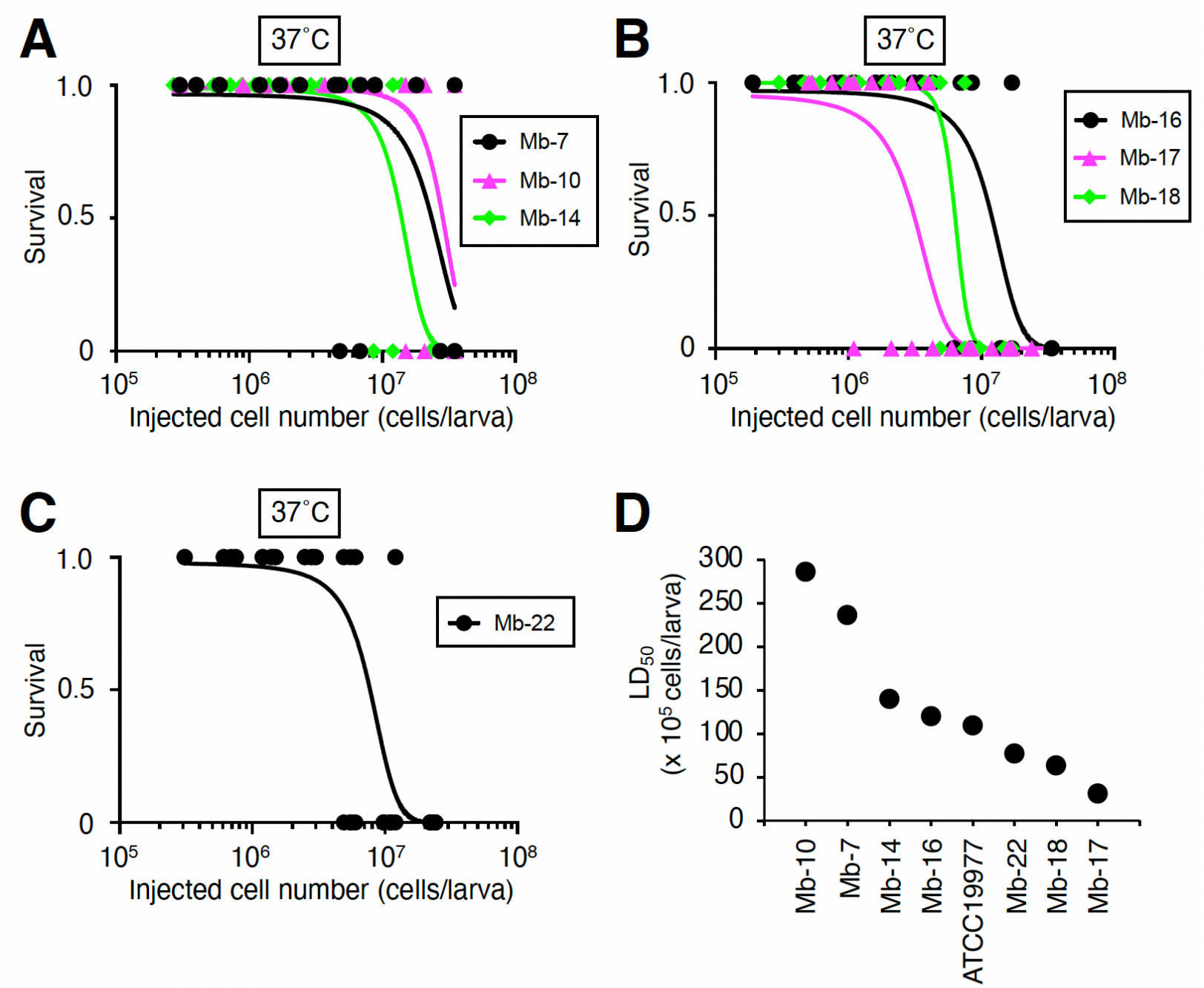

Figure 5. Comparison of pathogenicity among M. abscessus subsp. abscessus clinical isolates in a silkworm infection model

(A-C) Silkworms were injected with either saline, M. abscessus subsp. abscessus, Mb-7, Mb-10, Mb-14, Mb-16, Mb-17, Mb-18, or Mb-22 cell suspensions $\left(2 \times 10^{5}-3.5 \times 10^{7}\right.$ cells $/ 50 \mu \mathrm{l}$ ) and incubated at $37^{\circ} \mathrm{C}$. Live and dead silkworms are indicated as 1 and 0 , respectively. The curves are data from three independent experiments combined in a simple logistic regression model.

(D) A plot of $\mathrm{LD}_{50}$ values determined from $A-C$. 